\title{
The VAPB-PTPIP51 endoplasmic reticulum- mitochondria tethering proteins are present in neuronal synapses and regulate synaptic activity
}

Patricia Gómez-Suaga', Beatriz G. Pérez-Nievas', Elizabeth B. Glennon', Dawn H. W. Lau', Sebastien Paillusson', Gábor M. Mórotz', Tito Cali ${ }^{2}$, Paola Pizzo², Wendy Noble ${ }^{1}$ and Christopher C. J. Miller ${ }^{1 *}$ (D)

\begin{abstract}
Signaling between the endoplasmic reticulum (ER) and mitochondria regulates a number of key neuronal functions. This signaling involves close physical contacts between the two organelles that are mediated by "tethering proteins" that function to recruit regions of ER to the mitochondrial surface. The ER protein, vesicle-associated membrane protein-associated protein B (VAPB) and the mitochondrial membrane protein, protein tyrosine phosphatase interacting protein-51 (PTPIP51), interact to form one such tether. Recently, damage to ER-mitochondria signaling involving disruption of the VAPB-PTPIP51 tethers has been linked to the pathogenic process in Parkinson's disease, frontotemporal dementia (FTD) and related amyotrophic lateral sclerosis (ALS). Loss of neuronal synaptic function is a key feature of Parkinson's disease and FTD/ALS but the roles that ER-mitochondria signaling and the VAPB-PTPIP51 tethers play in synaptic function are not known. Here, we demonstrate that the VAPB-PTPIP51 tethers regulate synaptic activity. VAPB and PTPIP51 localise and form contacts at synapses, and stimulating neuronal activity increases ER-mitochondria contacts and the VAPB-PTPIP51 interaction. Moreover, siRNA loss of VAPB or PTPIP51 perturbs synaptic function and dendritic spine morphology. Our results reveal a new role for the VAPB-PTPIP51 tethers in neurons and suggest that damage to ER-mitochondria signaling contributes to synaptic dysfunction in Parkinson's disease and FTD/ALS.
\end{abstract}

Keywords: VAPB, PTPIP51, Endoplasmic reticulum, Mitochondria, Parkinson's disease, Frontotemporal dementia, Amyotrophic lateral sclerosis, Synapse

\section{Introduction}

Signaling between the ER and mitochondria regulates a variety of fundamental cellular processes. These include energy metabolism, $\mathrm{Ca}^{2+}$ homeostasis, phospholipid synthesis, mitochondrial biogenesis and trafficking, ER stress responses, autophagy and inflammation [7, 34, 37, 39]. This signaling is facilitated by close physical contacts between the two organelles such that up to approximately $20 \%$ of the mitochondrial surface is closely apposed $(10-30 \mathrm{~nm}$ distances) to ER membranes. These regions of ER are

\footnotetext{
* Correspondence: chris.miller@kcl.ac.uk

${ }^{1}$ Department of Basic and Clinical Neuroscience, Maurice Wohl Clinical Neuroscience Institute, Institute of Psychiatry, Psychology and Neuroscience, King's College London, London SE5 9RX, UK

Full list of author information is available at the end of the article
}

termed mitochondria associated ER membranes (MAM) [7, $34,37,39]$.

The mechanisms by which ER membranes are recruited to the mitochondrial surface are not fully understood but it is widely agreed that the process involves "tethering proteins" which act to scaffold and anchor the two organelles in close proximity. One such tether involves an interaction between the integral ER protein, vesicle-associated membrane protein-associated protein B (VAPB) and the outer mitochondrial membrane protein, protein tyrosine phosphatase interacting protein-51 (PTPIP51) [9, 45]. The VAPB-PTPIP51 tethers are now known to facilitate inositol 1,4,5-trisphosphate (IP3) receptor mediated delivery of $\mathrm{Ca}^{2+}$ from ER stores to mitochondria, mitochondrial ATP production and autophagy, all of which are known to be regulated by ER-mitochondria crosstalk $[9,13,33,45,46]$. 
The pivotal roles that ER-mitochondria signaling plays in so many important physiological functions suggest that damage to this signaling will have detrimental effects on cellular homeostasis. Indeed, perturbation of ER-mitochondria contacts and signaling is associated with the major human neurodegenerative diseases Alzheimer's disease, Parkinson's disease and FTD/ALS [1, 23, 24, 34]. Notably, disruption to the VAPB-PTPIP51 tethers has been linked to Parkinson's disease and FTD/ALS [9, 33, 45, 46]. FTD is the second most common form of presenile dementia after Alzheimer's disease and is now known to be clinically, genetically and pathologically linked to ALS, the most common form of motor neuron disease [24, 28].

Loss of synaptic function is a key pathogenic feature of Parkinson's disease and FTD/ALS. Indeed, synaptic loss underlies the cognitive and motor dysfunctions that characterise these disorders [3, 17, 43]. Both ER and mitochondria are known to be present in synaptic regions $[16,18,31,50]$. However, any role of the VAPB-PTPIP51 tethers and ER-mitochondria signaling in synaptic function is currently unknown. Such knowledge is essential not only for comprehending the normal roles of ER-mitochondria signaling in synaptic function, but also for determining any pathological role that disruption to the VAPB-PTPIP51 tethers might play in Parkinson's disease and FTD/ALS. Here, we show that VAPB and PTPIP51 are present and interact in synaptic regions, that their interactions are stimulated by neuronal activity, and that loss of VAPB and PTPIP51 disrupts synaptic function.

\section{Materials and methods}

\section{Plasmids and siRNAs}

SPLICS $_{\mathrm{s}}$ and SypHy-RGECO reporter plasmids were as described [6, 20]; pEGFPC1 was from Clontech. Verified non-targeting control and rat VAPB and PTPIP51 siRNAs were purchased from GE Healthcare Dharmacon (Accell range). Sequences were: VAPB A-091473-17\# 5'-GUGC UGUUCUUUAUUGUUG-3', A-091473-18\# 5' - CUUA UGGAUUCAAAACUUA-3', A-091473-19\# 5' -GGUUCA GUCUAUGUUUGCU-3', A-091473-20\# 5'-GUUACAGC CUUUCGAUUAU-3'; PTPIP51 (Fam82a2) A-092062-13\# 5' -CCUUUAAUGUCAUACCUUA-3', A-092062-14\# 5'-G CUUUAGCUUCAAGGAACA-3', A-092062-15\# 5' - GCU ACAGCCUUGUUUGAAA-3', A-092062-16\# 5' - CUCU GGACCUUGAUAUGGA-3'.

\section{Antibodies and chemicals}

Rabbit and rat antibodies to VAPB and PTPIP51 were as described [9]. Rabbit anti-PTPIP51 and chicken anti-MAP 2 were from Gentex. Rabbit anti-translocase of the outer mitochondrial membrane protein-20 (TOM20), mouse anti-glyceraldehyde 3-phosphate dehydrogenase (GAPDH) and goat anti-synaptophysin were from Santa Cruz
Biotechnology. Mouse anti- $\beta$-Tubulin Isotype III antibody and mouse anti- $\beta$-Actin were from Sigma. Mouse anti-post synaptic density protein-95 (PSD95) was from Millipore, mouse anti-protein disulphide isomerase (PDI) was from Thermo Fisher Scientific and mouse anti-phosphorylated neurofilament heavy chain (NFH) (antibody SMI31) was from Sternberger Monoclonals Inc. Species specific goat and donkey anti-mouse, -rabbit and -chicken Igs coupled to AlexaFluor-488, - 594 or - 647 were from Invitrogen, Jackson ImmunoResearch, ThermoFisher or Abcam. FM 4-64 was from Invitrogen, DL-2-amino-5-phosphonovaleric acid (AP5) was from Cayman chemical company and 6-cyano-7-nitroquinoxaline-2,3-dione (CNQX) was from Santa Cruz.

\section{Hippocampal neuronal culture and transfection}

Hippocampal neurons were obtained from embryonic day 18 rat embryos and cultured in Neurobasal medium containing B27 supplement, $100 \mathrm{IU} / \mathrm{ml}$ penicillin, $100 \mu \mathrm{g} / \mathrm{ml}$ streptomycin and $2 \mathrm{mM} \mathrm{L}$-glutamine (Invitrogen). Neurons were cultured on poly-d-lysine-coated glass cover slips in 12-well plates and analysed at DIV20-23. For siRNA studies, neurons were untreated or treated with $1 \mu \mathrm{M}$ of each siRNA for $72 \mathrm{~h}$ prior to analyses. For transfection studies, neurons were transfected at DIV5 using Lipofectamine 2000 (Invitrogen) $(0.5 \mu \mathrm{g}$ plasmid DNA, $1 \mu \mathrm{l}$ Lipofectamine 2000 per well) according to the manufacturer's instructions.

\section{Protein fractionation, SDS-PAGE and immunoblotting}

Cells were harvested for sodium dodecyl sulphate-polyacrylamide gel electrophoresis (SDS-PAGE) by washing with calcium-free phosphate buffered saline (PBS) pre-warmed at $37^{\circ} \mathrm{C}$ and scraping into SDS-PAGE sample buffer containing protease inhibitors (Complete Roche), $1 \mathrm{mM} \mathrm{Na} \mathrm{mO}_{4}$ and $5 \mathrm{mM} \mathrm{NaF}$. Samples were then heated for $10 \mathrm{~min}$ at $100{ }^{\circ} \mathrm{C}$, sonicated and centrifuged at $10000 \mathrm{~g}$ (av) for $10 \mathrm{~min}$. Total, cytosolic and synaptoneurosome proteins were prepared from rat brains essentially as described [35, 47]. Protein concentrations were determined using a commercial BCA assay (Pierce). Samples were prepared for SDS-PAGE by addition of sample buffer and then resolved on 10 or $15 \%$ SDS-PAGE gels and transferred to nitrocellulose membranes (Schleicher \& Schuell Bioscence) by wet electroblotting (BioRad). Membranes were blocked with Tris-HCl-buffered saline (TBS) containing 5\% dried milk and $0.1 \%$ Tween-20 for $1 \mathrm{~h}$ at $20^{\circ} \mathrm{C}$, and then incubated with primary antibodies in blocking buffer for $16 \mathrm{~h}$ at $4{ }^{\circ} \mathrm{C}$. Following washing in TBS containing $0.1 \%$ Tween-20, the blots were incubated with horseradish peroxidase conjugated secondary antibodies and developed using chemiluminescence with a Luminata Forte Western HRP substrate system according to the manufacturer's 
instructions (Millipore). Chemiluminescence signals were detected using a BioRad ChemiDoc MP Imaging system.

Immunofluorescence staining and proximity ligation assays Neurons grown on coverslips were fixed for $15 \mathrm{~min}$ at $20{ }^{\circ} \mathrm{C}$ with $4 \%(w / v)$ paraformaldehyde in PBS and then permeabilized with PBS containing 0.5\% Triton X-100 for $15 \mathrm{~min}$. Samples were then preincubated with blocking buffer (PBS containing 10\% goat or 2\% donkey serum and $0.5 \%$ Triton $\mathrm{X}-100$ ) for $1 \mathrm{~h}$ and incubated with primary antibodies diluted in blocking buffer for $16 \mathrm{~h}$ at $4{ }^{\circ} \mathrm{C}$. Following washing in PBS containing $0.5 \%$ Triton X-100, the samples were incubated with goat/ donkey anti-rabbit, mouse, rat or chicken Igs coupled to AlexaFluor -488 , -594 or 647 in PBS for $1 \mathrm{~h}$, washed in PBS and then mounted in Vectashield mounting medium (Vector Laboratories). Proximity ligation assays (PLAs) to identify the VAPB-PTPIP51 interaction were performed essentially as described previously using Duolink reagents (Sigma-Aldrich) [13]. Briefly, neurons were fixed in $4 \%$ paraformaldehyde in PBS and probed with rat anti-PTPIP51 and rabbit anti-VAPB antibodies, and signals developed using a Duolink In Situ Orange kit (Sigma-Aldrich). Following PLAs, neurons were immunolabeled for synaptophysin and PSD95.

\section{Microscopy}

Super resolution structured illumination microscopy (SIM) was performed using Nikon Eclipse Ti-E Inverted microscopes with $100 \times 1.49$ NA CFI objectives and equipped with Nikon N-SIM or Visitech iSIM Super Resolution Systems. Images were captured using an Andor iXon EMCCD camera and reconstructed using Nikon imaging software Elements Advanced Research with N-SIM module or Nikon deconvolution software for iSIM.

Live cell imaging was performed by time-lapse microscopy using a Nikon Eclipse Ti microscope equipped with an Intenslight C-HGFI light source, CFI Apo Lambda S 60x/1.40 objective, TiND6 PFS-S Perfect Focus Unit and EGFP, DsRed and EGFP/DsRed dual filter sets (Chroma Technology). Images were captured using an Andor Neo sCMOD camera. For dual imaging, EGFP and DsRed signals were captured simultaneously using an EGFP/ DsRed dual filter set, an Andor TuCam camera adapter system equipped with an emission GFP/RFP dichroic filter set and two Andor Neo sCMOD cameras. Movements were recorded using Nikon NIS-Elements AR software at 2 or $3 \mathrm{~s}$ time-lapse intervals. Temperature was maintained at $37^{\circ} \mathrm{C}$ using a microscope incubation chamber (Solent Scientific). During recordings, neurons were kept under constant perfusion $(0.5 \mathrm{ml} / \mathrm{min})$ with external solution using a Bio-Logic MSC200 fast perfusion system. External solution comprised $140 \mathrm{mM} \mathrm{NaCl}$, $5 \mathrm{mM} \mathrm{KCl}, 5 \mathrm{mM} \mathrm{NaHCO}, 1 \mathrm{mM} \mathrm{MgCl}_{2}, 1.2 \mathrm{mM}$
$\mathrm{CaCl}_{2}, 1.2 \mathrm{mM} \mathrm{Na} \mathrm{HPO}_{4}, 10 \mathrm{mM}$ glucose in $20 \mathrm{mM}$ HEPES buffer $\mathrm{pH} 7.4$.

Electrical field stimulations were performed in a Chamlide EC-B18 field stimulation chamber and field stimulation (25 mA pulses of $1 \mathrm{~ms}$ duration) delivered by an Isolated Stimulator DS3 controlled by a Train/Delay Generator DG2A (Digitimer). Analyses involving FM 4-64 were performed essentially as described [19]. Briefly, FM 4-64 $(2.5 \mu \mathrm{M})$ was added in external solution and loaded into neurons with electrical field stimulation $(20 \mathrm{~Hz}$ for $60 \mathrm{~s}$ ) which was applied with an insert adaptor in the culture plates (RC-37FS, Warner instruments). FM 4-64 dye was removed from the surface membranes by incubation in external solution containing NMDA and AMPA receptor antagonists $(50 \mu \mathrm{M}$ AP5, $10 \mu \mathrm{M}$ CNQX) followed by incubation in $\mathrm{Ca}^{2+}$-free external solution. Neurons were then transferred to the microscope field stimulation chamber and analysed in time-lapse. For analyses of SPLICS stimulations were delivered at frequency of $30 \mathrm{~Hz}$ for $10 \mathrm{~s}$. For analyses of FM 4-64, 3 field stimulations of $60 \mathrm{~s}$ at 20 $\mathrm{Hz}$ were applied at $60 \mathrm{~s}$ intervals as described [19]. For analyses of SypHy-RGECO signals, 3 field stimulations of $10 \mathrm{~s}$ at $30 \mathrm{~Hz}$ were applied at $60 \mathrm{~s}$ intervals essentially as described [20]. VAPB-PTPIP51 PLA field stimulations were conducted in the culture plates using the insert adaptor delivering stimulations of $30 \mathrm{~Hz}$ for $10 \mathrm{~s}$. Neurons were then fixed and processed for PLAs and immunostaining.

Confocal microscopy images were acquired using a Leica TCS-SP5 confocal microscope using a 63x HCX PL APO lambda blue CS 1.4 oil UV objective. Z-stack images were analysed and processed using Leica Applied Systems (LAS AF6000) image acquisition software.

PLA signals were quantified in close proximity (less than $1 \mu \mathrm{m})$ to synaptic contacts identified by synaptophysin/ PSD95 apposition using NIH ImageJ in $20 \mu \mathrm{m}$ segments of dendrites after the first dendritic branchpoint. ER-mitochondria contacts were quantified by analyses of PDI/ TOM20 colocalization with Pearson's coefficient using Nikon Imaging Software Elements AR. Dendritic spine densities were quantified using NeuronStudio software (CNIC). Active spines involving apposition of spines to synaptophysin signals were quantified using Image in $20 \mu \mathrm{m}$ segments of dendrites located after the first branch. Time-lapse movies were processed offline using the NIS-Elements AR software and ImageJ. FM 4-64 and SypHy-RGECO signals were quantified as described $[19,20]$.

\section{Results}

\section{VAPB and PTPIP51 localise and interact at synapses}

We used super resolution structured illumination microscopy (SIM) to study whether VAPB and PTPIP51 localise to synaptic regions in cultured 20-23 day in vitro (DIV) rat hippocampal neurons; these neurons form mature functional synapses. We first stained neurons for 
synaptophysin+PSD95 and either VAPB or PTPIP51. Synaptophysin and PSD95 are pre- and postsynaptic markers and synaptic pairs can be identified by close apposition (less than $0.5 \mu \mathrm{m}$ ) of these proteins [36]. These studies revealed that both VAPB and PTPIP51 localise in close proximity to synaptic pairs (Fig. 1a, b). We then enquired whether this synaptic localisation was near presynaptic axonal boutons and/or postsynaptic dendritic spines. To identify VAPB and PTPIP51 in presynaptic regions, neurons were immunostained for phosphorylated NFH to identify axons, synaptophysin and either VAPB or PTPIP51. To identify VAPB and PTPIP51 in postsynaptic regions, neurons were immunostained for microtubule-associated protein-2 (MAP2) to identify dendrites, PSD95 and either VAPB or PTPIP51. These studies revealed that both VAPB and PTPIP51 were present in close proximity to both axonal boutons and dendritic spines (Fig. 1c, d).

To complement these studies, we prepared biochemical fractions of synaptoneurosomes from rat brain and probed these on immunoblots for VAPB and PTPIP51, synaptophysin and PSD95 as synaptic markers, and PDI and TOM20 as ER and mitochondrial markers. Such synaptoneurosome preparations have been extensively characterised and contain the presynaptic synaptosome with attached postsynaptic neurosome [35, 47]. For these immunoblots we also loaded equal amounts of total mouse brain and cytosolic (soluble) proteins for comparison. These studies revealed that VAPB and PTPIP51 were present in synaptoneurosomes (Fig. 1e). Finally, we performed proximity ligation assays (PLAs) to determine whether VAPB and PTPIP51 are closely associated with each other in synaptic regions in the cultured hippocampal neurons. The distances required for PLA signals are similar to those detected by resonance energy transfer between fluorophores (i.e. approximately $10 \mathrm{~nm}$ ) and so PLAs are suitable for quantifying ER-mitochondria contacts of 10-30 nm [42]. Indeed, PLAs including ones for VAPB and PTPIP51 have already been used to quantify ER-mitochondria contacts [9, 13, 16, 33, 46]. Synapses were identified by close apposition of synaptophysin and PSD95 immunofluorescent signals. These studies revealed that VAPB-PTPIP51 PLA signals were closely associated with synapses (less than $1 \mu \mathrm{m}$ distance) (Fig. 1f) (and see Fig. 2c). Thus, VAPB and PTPIP51 localise and interact in synaptic regions.

\section{Synaptic activity stimulates ER-mitochondria contacts and the VAPB-PTPIP51 interaction}

The localisation and interaction of VAPB and PTPIP51 in synaptic regions suggest they play a role in synaptic function. We therefore monitored how synaptic activity affects ER-mitochondria contacts and the VAPBPTPIP51 interaction. To do so we first utilised split green fluorescent protein (GFP) ER-mitochondria contact reporter (SPLICS) plasmids that comprise outer mitochondrial membrane and ER targeting sequences fused respectively to the GFP1-10 and $\beta$-strand 11 of the superfolder GFP variant [6]. This SPLICS $_{\mathrm{s}}$ contact sensor only fluoresces when the two organelles are brought into close (approximately $10 \mathrm{~nm}$ ) proximity so as to restore GFP functional domains [6]. SPLICS transfected hippocampal neurons displayed fluorescence signals in cell bodies, axons and dendrites demonstrating close ER-mitochondria contacts in the cells (Fig. 2a). The intensities of these SPLICS ${ }_{\mathrm{s}}$ signals displayed no noticeable changes over extended monitoring periods $(10 \mathrm{~min})$ which indicates that ER-mitochondria contacts are stable in the neurons (Fig. 2b). This observation is consistent with previous studies of SPLICS $_{\mathrm{s}}$ transfected cells which show stable signals over many hours [6]. However, induction of synaptic activity by electrical field stimulation $[19,20]$ markedly increased the SPLICS $\mathrm{s}$ signals in cell bodies and processes (Fig. 2c and Additional file 1: Movie 1).

To determine whether any of these increased SPLICS fluorescent signals were associated with active synapses, we loaded the neurons with the red fluorescent synaptic vesicle recycling dye FM 4-64 and monitored both FM 4-64 and SPLICS s fluorescent signals prior to and following electrical field stimulation. During loading, FM 4-64 is taken into synaptic vesicles as they form via endocytosis; however, during synaptic activity FM 4-64 fluorescent signals are reduced as vesicles are released from active synapses [19]. Electrical field stimulation induced selective decreases in FM 4-64 signals of approximately $30 \%$; these reductions are in line with those reported by others following similar treatments $[27,30$, 48] (Fig. 2d). These decreases were accompanied by increased SPLICS ${ }_{\mathrm{s}}$ fluorescence indicating increased ER-mitochondria contacts (Fig. 2d). Notably, many of these increased SPLICS signals localised close to active synapses as identified by reduction of FM 4-64 signals (Fig. 2d and Additional file 2: Movie 2).

We also enquired whether the increases in ER-mitochondria contacts induced by synaptic activity in the hippocampal neurons were linked to changes in the VAPB-PTPIP51 interaction. To do so, we performed VAPB-PTPIP51 PLAs on unstimulated neurons and on neurons following electrical field stimulation, and monitored whether any changes in PLA signals were close to synapses. Synapses were identified by apposition of synaptophysin and PSD95 immunofluorescent signals. Quantification of these PLA signals were in the same regions of unstimulated and stimulated neurons $(20 \mu \mathrm{m}$ segments after the first dendritic branchpoint). Electrical field stimulation increased the numbers of VAPB-PTPIP51 PLA signals and this included signals that were close (less than $1 \mu \mathrm{m}$ distance) to synapses (Fig. 2e). 
a
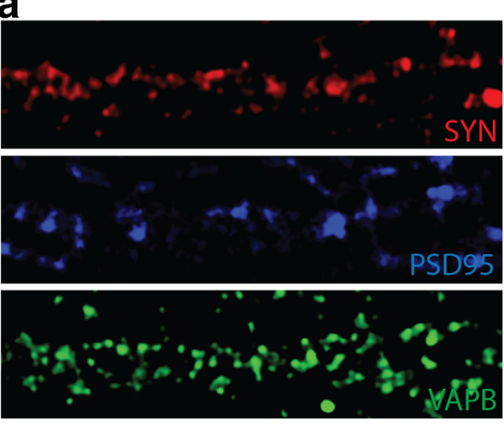

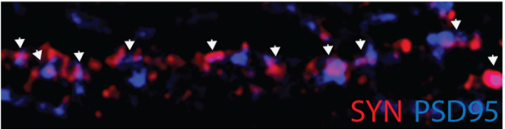

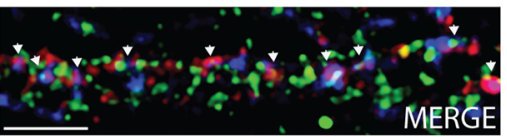

c
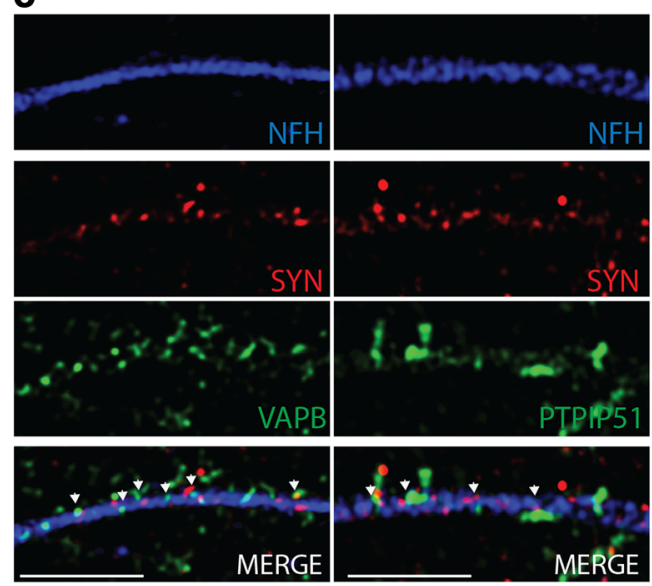

MERGE

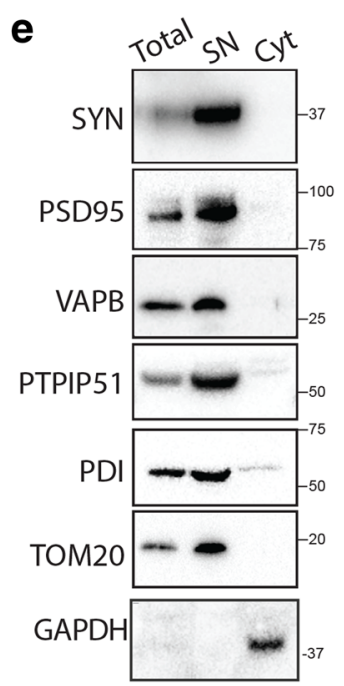

f
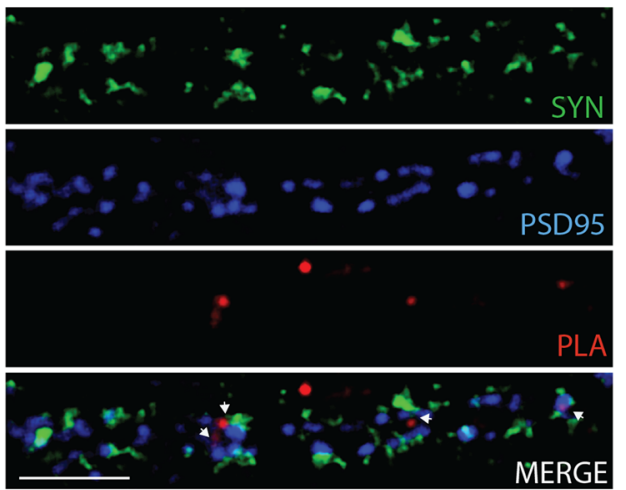

d
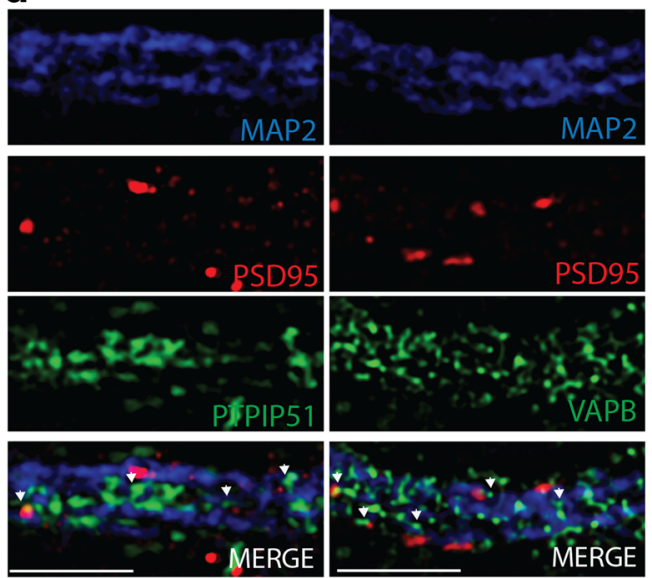

MERGE

Fig. 1 (See legend on next page.) 
(See figure on previous page.)

Fig. 1 VAPB and PTPIP51 localise and interact at synapses. $\mathbf{a}$ and $\mathbf{b}$ Super-resolution SIM images of VAPB (a) and PTPIP51 (b) localisation close to synaptic pairs. Hippocampal neurons were immunolabeled for synaptophysin (SYN) and PSD95, and either VAPB or PTPIP51. SYN + PSD95 identifies synaptic contacts (arrows) via apposition of labelling. MERGE images show VAPB or PTPIP51 staining closely localised to synaptic contacts indicated by arrows shown in SYN + PSD95. $\mathbf{c}$ and $\mathbf{d}$ SIM images of VAPB and PTPIP51 localisation close to presynaptic (c) and postsynaptic (d) compartments. c shows presynaptic compartment identified by immunostaining for axons with phosphorylated NFH and synaptophysin. $\mathbf{d}$ shows postsynaptic compartment identified by immunostaining for dendrites with MAP2 and PSD95. Arrows in (c) and (d) MERGE indicate some VAPB and PTPIP51 labeling close to synaptophysin and PSD95. e VAPB and PTPIP51 are present in synaptoneurosomes. Immunoblot shows equal loading $(12 \mu \mathrm{g})$ of total mouse brain protein, synaptoneurosome (SN) and soluble cytoplasmic protein (Cyt) fractions probed for synaptophysin (SYN), PSD95, VAPB, PTPIP51, PDI (ER marker) TOM20 (mitochondrial marker) and GAPDH (cytosolic marker). f VAPB-PTPIP51 PLA signals localise close to synapses in hippocampal neurons. PLAs were performed and the samples then immunostained for synaptophysin (SYN) and PSD95 to identify synapses. Arrows in MERGE indicate VAPB-PTPIP51 PLA signals close to synaptic contacts. Scale bars in a-d and $\mathrm{f}=2 \mu \mathrm{m}$

\section{Loss of VAPB and PTPIP51 reduce dendritic spine numbers and synaptic activity}

The above findings suggest that the VAPB-PTPIP51 tethers play a role in synaptic function. To test this possibility further, we first enquired how siRNA loss of VAPB or PTPIP51 affects dendritic spine numbers in the hippocampal neurons since spine numbers are closely linked with synapse function [21]. siRNAs for VAPB and PTPIP51 have been characterised previously $[9,13,45]$ and we confirmed that these siRNAs reduced VAPB and PTPIP51 protein levels in the rat neurons (Fig. 3a). Individual VAPB/PTPIP51 siRNAs were all effective as were the mixed "pools" of these siRNAs. The pooled siRNAs led to approximate $82 \%( \pm 9.1 \%)$ and $66 \%( \pm 2.9 \%)$ reductions in VAPB and PTPIP51 levels respectively, and were used in all later experiments. We also confirmed that as in other cell types, this loss of VAPB and PTPIP51 reduced ER-mitochondria contacts in the neurons by performing super resolution SIM on untreated, control, VAPB and PTPIP51 siRNA treated neurons that were immunostained for PDI and TOM20 to label ER and mitochondria respectively. As predicted, loss of VAPB or PTPIP51 both reduced ER-mitochondria contacts in the neurons (Fig. 3b).

To determine how siRNA loss of VAPB or PTPIP51 affects dendritic spine numbers, we transfected neurons with EGFP to reveal neuronal morphology and identify dendrites; such approaches have been used in many other studies e.g. $[8,11,49]$. We then quantified spine numbers in the same dendritic regions of the different treated neurons $(20 \mu \mathrm{m}$ segments after the first branchpoint). Loss of VAPB or PTPIP51 reduced spine numbers (Fig. 3c). We also determined how loss of VAPB/PTPIP51 affected spines that are part of active synaptic pairs by immunostaining the EGFP transfected neurons for synaptophysin. Apposition of spines with synaptophysin immunolabelling can be used to identify active spines [49]. Loss of VAPB or PTPIP51 also reduced active spine numbers (Fig. 3d).

Since morphological changes in synapses are linked to synaptic function, we next studied how loss of VAPB or PTPIP51 affects synaptic activity. We first monitored release of pre-loaded FM 4-64 dye following electrical field stimulation in control, VAPB and PTPIP51 siRNA knockdown neurons. As shown above (Fig. 2d) and by others [19], electrical field stimulation induced release of FM 4-64 from synapses with concomitant decreases in dye signals. However, siRNA knockdown of VAPB or PTPIP51 inhibited this loss of FM 4-64 signal (Fig. 4a).

We also utilised a genetic indicator reporter plasmid that permits imaging of both presynaptic $\mathrm{Ca}^{2+}$ influx and vesicle exocytosis. This reporter (SypHy-RGECO) involves fusion of the synaptic vesicle protein synaptophysin to both a red shifted $\mathrm{Ca}^{2+}$ indicator (RGECO1) and a GFP-based $\mathrm{pH}$ sensor (pHluorin). This enables optical correlates of $\mathrm{Ca}^{2+}$ and $\mathrm{pH}$ changes to be simultaneously monitored in synaptic vesicles [20]. Due to the fixed stoichiometry of the two probes, the ratio of the two responses can be used to provide an optical correlate of the $\mathrm{Ca}^{2+}$ dependence of vesicle release and so provide a measure of presynaptic activity [20]. Hence, the correct way of reporting data obtained from the SypHy-RGECO indicator is to display the ratio of SypHy and RGECO signals (SypHy/RGECO) [20]. SypHy-RGECO transfected neurons displayed punctate fluorescent signals in both channels and electrical field stimulation induced increases in these signals as described by others [20] (Fig. 4b). However, compared to control cells, the ratio of SypHy/ RGECO amplitudes of these signals were reduced in both VAPB and PTPIP51 siRNA treated cells consistent with diminished presynaptic activity (Fig. 4c). Thus, loss of VAPB and PTPIP51 reduces dendritic spine and active spine numbers, and decreases synaptic activity following electrical field stimulation.

\section{Discussion}

Loss of synapses and synaptic dysfunction are principal features of the major human neurodegenerative diseases Alzheimer's disease, Parkinson's disease and FTD/ALS [3, 17, 43]. Damage to ER-mitochondria contacts and signaling is increasingly linked to these diseases and for Parkinson's disease and FTD/ALS this includes disruption to the VAPB-PTPIP51 ER-mitochondria tethering proteins $[1,23,33,34,45,46]$. However, whilst ER and mitochondria are both present in synaptic regions $[16$, $18,31,50$ ] any role that the VAPB-PTPIP51 tethers play in synaptic function is not currently known. Such knowledge is essential for determining whether disruption to 
a

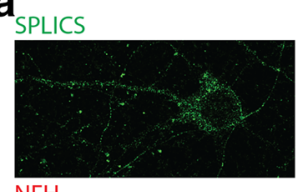

$\mathrm{NFH}$

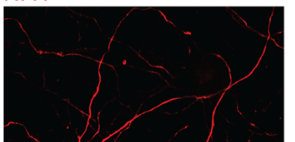

MAP2

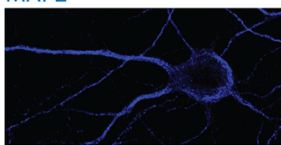

MERGE

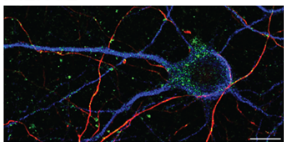

d

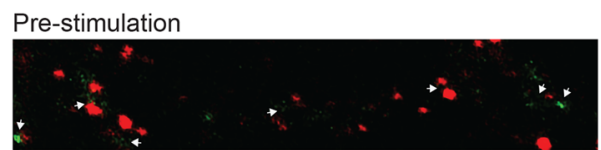

Post-stimulation (300s)
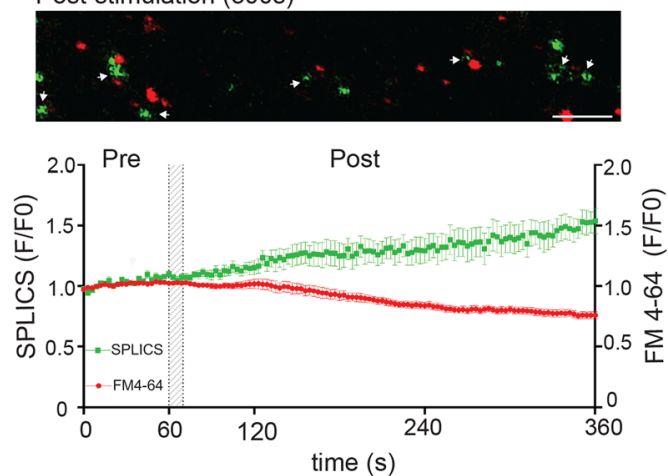

c

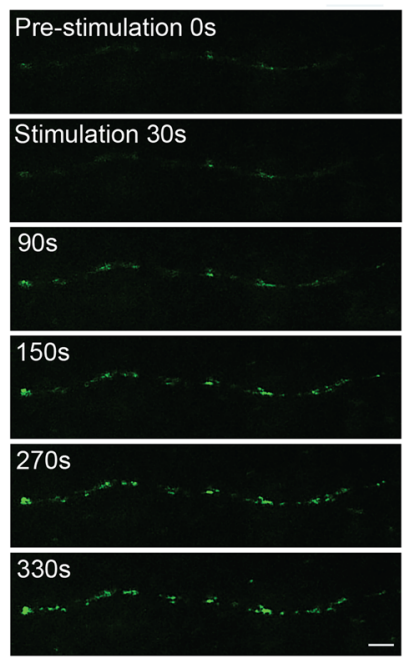

e PLAs Syn PSD95
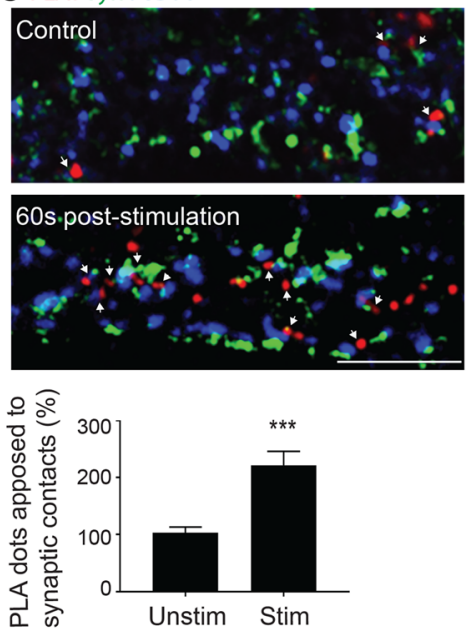

Fig. 2 Synaptic activity regulates ER-mitochondria contacts and the VAPB-PTPIP51 interaction in hippocampal neurons. a Confocal z-stack of neuron

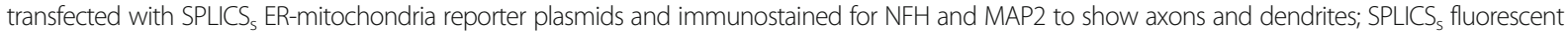

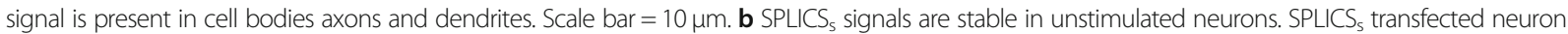
axons were imaged in time-lapse over a $10 \mathrm{~min}$ period and displayed no significant changes in SPLICS signals. Graph shows average fluorescence signal F/initial signal F0 as a \%. Error bars are SEM; $N=14$ neurons from 8 different cultures. (c and d), Electrical field stimulation of synaptic activity increases ER-

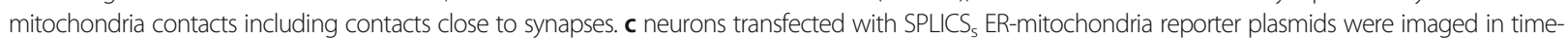

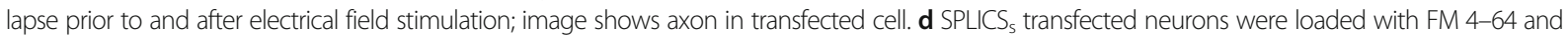
SPLICS $_{5}$ (green) and FM 4-64 (red) signals imaged in time-lapse prior to and after electrical field stimulation. Arrows indicate increased SPLICS signals closely associated with active synapses identified by loss of FM 4-64 signal. Graph shows changes in fluorescence signal (fluorescence at each time-point (F)/ average of all pre-stimulation frames (FO)) from 7 synapses. Error bars are SEM. Analyses of SPLICS signal reveals no changes prior to stimulation in agreement with data shown in (b) above but significant increases following stimulation (time 30-60 s; prior to stimulation v time 300-360; post stimulation $p \leq 0.0001$, one way ANOVA with Tukey's post hoc test). e Representative SIM image of VAPB-PTPIP51 PLA signals in synaptic regions of unstimulated neurons and in neurons after (60 s) electrical field stimulation. VAPB-PTPIP51 PLAs (red) were performed and neurons then immunostained for synaptophysin (green) and PSD95 (blue). Arrows indicate PLA signals close (less than $1 \mu \mathrm{m}$ ) to synapses as identified by apposition of synaptophysin and PSD95 signals. Bar chart shows normalised VAPB-PTPIP51 PLA signals (\%) close to synapses in unstimulated neurons and in neurons after electrical field stimulation. Data were analysed by Student's t test. $N=36$ neurons unstimulation and 34 neurons post stimulation from 3 independent experiments. Error bars are SEM; ${ }^{* * *} p \leq 0.001$. Scale bars in $\mathbf{c}, \mathbf{d}$ and $\mathbf{e}=2 \mu \mathrm{m}$ 

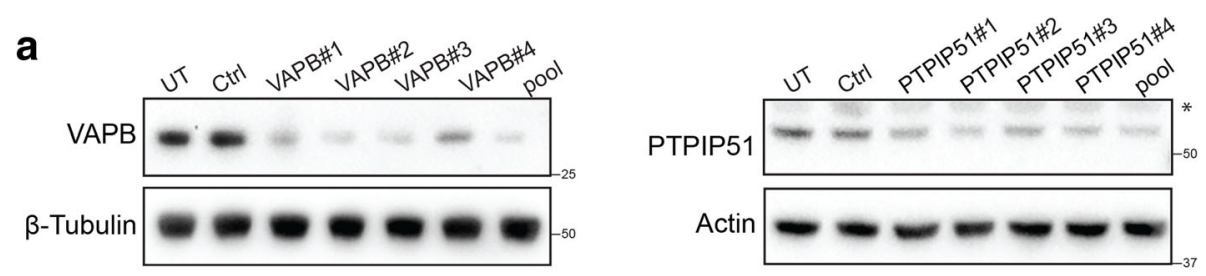

\section{b}
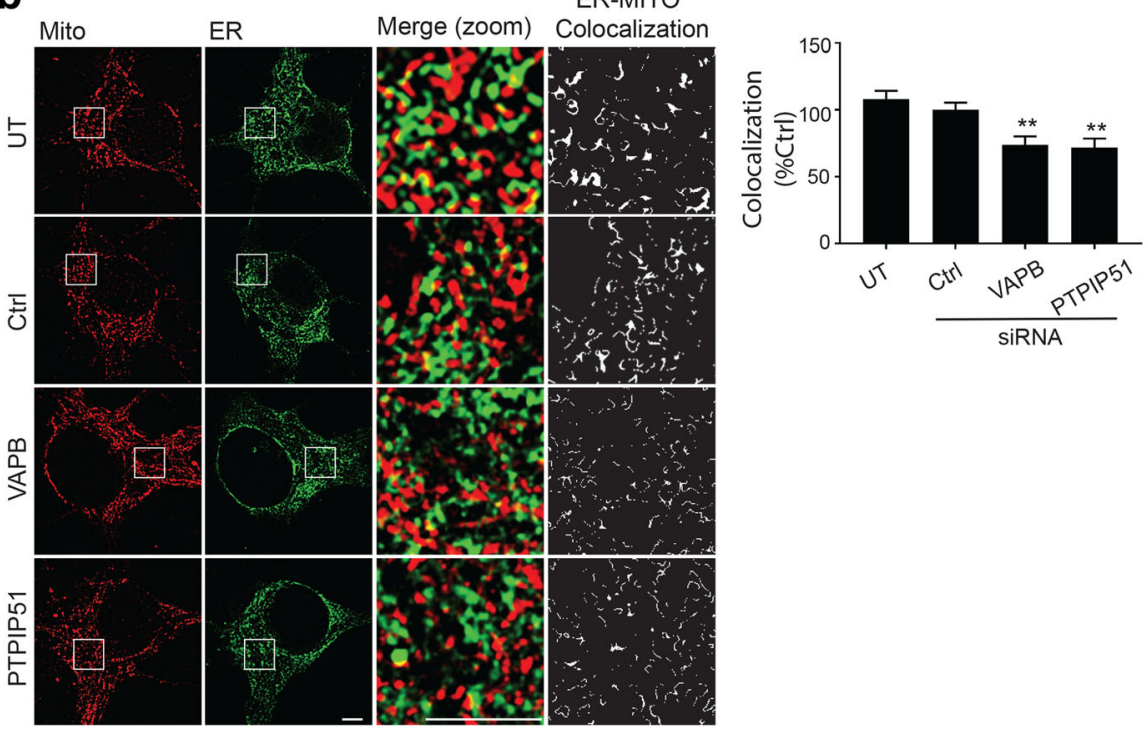

c

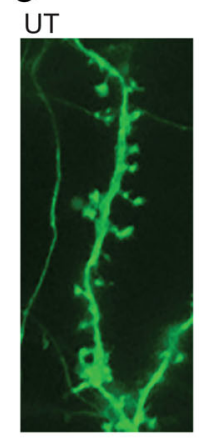

Ctrl
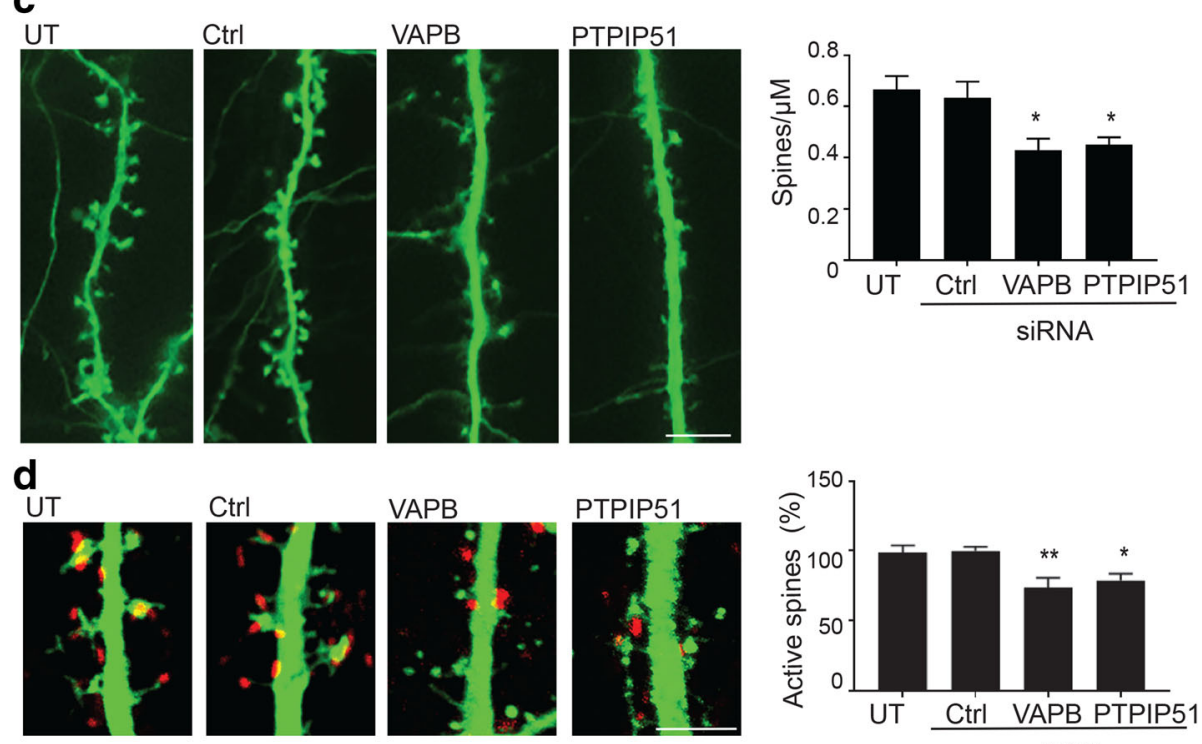

Fig. 3 (See legend on next page.)

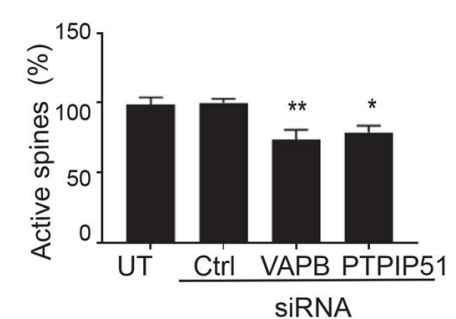


(See figure on previous page.)

Fig. 3 Loss of VAPB and PTPIP51 disrupts ER-mitochondria contacts and reduces dendritic spine and active spine numbers in hippocampal neurons. a Immunoblots showing VAPB and PTPIP51 levels in hippocampal neurons either untreated (UT) or treated with control (Ctrl), 4 different VAPB/PTPIP51 siRNAs or a pool of these siRNAs. PTPIP51 migrates at approximately $61 \mathrm{kD}$ in agreement with previous studies $[9,45]$. The weakly staining upper minor species $(*)$ on the PTPIP51 immunoblot does not display any consistent changes in response to the PTPIP51 siRNAs and so we believe it to be non-specific protein. We did not detect any other PTPIP51 species in the neurons. b Super resolution SIM images of neurons either untreated or treated with control, VAPB or PTPIP51 pooled siRNAs, and then immunostained for ER and mitochondria with PDI and TOM20 antibodies. Zooms are of boxed regions with merge and co-localisation of signals. Scale bars $=5 \mu \mathrm{m}$. Bar chart shows ER-mitochondria co-localisation normalized to control siRNA in the different samples. Data were analysed by oneway ANOVA with Tukey's post hoc test. $N=19$ neurons UT, $N=42$ neurons Ctrl siRNA, $N=42$ neurons VAPB siRNA, $N=40$ neurons PTPIP51 siRNA from 3 independent experiments; error bars are $\mathrm{SEM}^{* *} p \leq 0.01$. $\mathbf{c}$ Representative images of dendritic spines in EGFP transfected neurons either untreated or treated with control, VAPB or PTPIP51 siRNAs. Bar chart shows spine densities (spines $/ \mu \mathrm{m}$ ). Data were analysed by one-way ANOVA with Tukey's post hoc test. $N=14$ neurons UT, $N=21$ neurons Ctrl siRNA, N=17 neurons VAPB siRNA, N=21 neurons PTPIP51 siRNA from 3 independent experiments; error bars are SEM, ${ }^{*} p \leq 0.05$. Scale bar $=5 \mu \mathrm{m}$. d Representative images of active dendritic spines in EGFP transfected neurons either untreated or treated with control, VAPB or PTPIP51 siRNAs. Active spines were identified by immunostaining for the presynaptic marker synaptophysin (red) and monitoring spine and synaptophysin apposition. Bar chart shows \% of active spines normalised to control siRNA treatment. Data were analysed by one-way ANOVA with Tukey's post hoc test. $N=19$ neurons UT, $N=20$ neurons Ctrl siRNA, $N=22$ neurons VAPB siRNA, $N=27$ neurons PTPIP51 siRNA from 3 independent experiments; error bars are SEM, ${ }^{*} p \leq 0.05,{ }^{* *} p \leq 0.01$. Scale bar $=3 \mu \mathrm{m}$

ER-mitochondria signaling and the VAPB-PTPIP51 tethers contributes to synaptic dysfunction in Parkinson's disease and FTD/ALS.

Here we show that the VAPB-PTPIP51 ER-mitochondria tethers are present and interact at synapses. We also demonstrate that stimulating synaptic activity increases ER-mitochondria contacts in synaptic regions and that this involves increased interactions between VAPB and PTPIP51. Finally, we show that siRNA loss of VAPB or PTPIP51 to reduce ER-mitochondria contacts inhibits synaptic activity including alterations to synaptic vesicle release and dendritic spine numbers. Together, our results demonstrate that ER-mitochondria contacts mediated by the VAPB-PTPIP51 tethers regulate synaptic function.

To determine how VAPB and PTPIP51 siRNA loss affects presynaptic function, we utilised two experimental approaches. The first involved the synaptic vesicle recycling dye FM 4-64 which is loaded into synaptic vesicles as they form via endocytosis and then released following induction of synaptic activity [19]. Loss of VAPB and PTPIP51 both reduced FM 4-64 release consistent with inhibition of presynaptic activity. The initial loading of FM 4-64 requires stimulation of synaptic activity and so it would be interesting in future studies to determine whether loss of VAPB and PTPIP51 affects this process. Such studies would assist in determining whether the VAPB-PTPIP51 tethers affect synaptic vesicle endocytosis and recycling.

The second approach utilised a genetic indicator SypHy-RGECO that involves fusion of the synaptic vesicle protein synaptophysin to both a red shifted $\mathrm{Ca}^{2+}$ indicator (R-GECO1) and a GFP-based $\mathrm{pH}$ sensor (pHluorin). Synaptic activity induces increased $\mathrm{Ca}^{2+}$ levels and also changes in $\mathrm{pH}$ which are the result of release of neurotransmitter from the acidic synaptic vesicle into the more basic synaptic cleft. Since the pHluorin sensor is more active in basic conditions, stimulation of synaptic activity generates increases in both R-GECO1 and pHluorin signals. The SypHy-RGECO indicator therefore enables optical correlates of $\mathrm{Ca}^{2+}$ and $\mathrm{pH}$ changes to be simultaneously monitored in synaptic vesicles. As was the case with FM 4-64 experiments, loss of VAPB and PTPIP51 both inhibited presynaptic activity in assays involving SypHy-RGECO.

As detailed above, following their release, synaptic vesicles are endocytosed for re-cycling and this involves their re-acidification. Following stimulation of synaptic activity, pHluorin signals initially increase with synaptic vesicle release but then decrease as the vesicles are endocytosed. Over the times analysed in our experiments, we observed the expected increase in pHluorin signals following induction of synaptic activity but no marked decreases. However, the times taken for these decreases are variable and dependent firstly upon endocytosis rates and then the times taken for re-acidification of vesicles by vATPase proton pumps. The kinetics are also dependent upon experimental conditions such as the strength of electrical stimulation used to induce synaptic activity [38] and the type and strength of buffer used to bathe the neurons; stronger buffers require longer to acidify [2]. Finally, different indicator plasmids (e.g. fusion of pHluorin to synaptophysin, synaptobrevin and VGLUT1) can provide differences in rates of endocytosis. Interestingly, like us others have shown that the SypHy-RGECO indicator plasmid we use generates relatively stable high signals following electrical field stimulation [20]. Future studies that involve analyses of SypHy-RGECO signals at later time points will help determine how the SypHy-RGECO indicator responds to vesicle recycling.

Aside from these presynaptic effects, we also found that siRNA loss of VAPB and PTPIP51 decreased total dendritic spine numbers and also the numbers of active spines as determined by their apposition to presynaptic synaptophysin. The VAPB-PTPIP51 

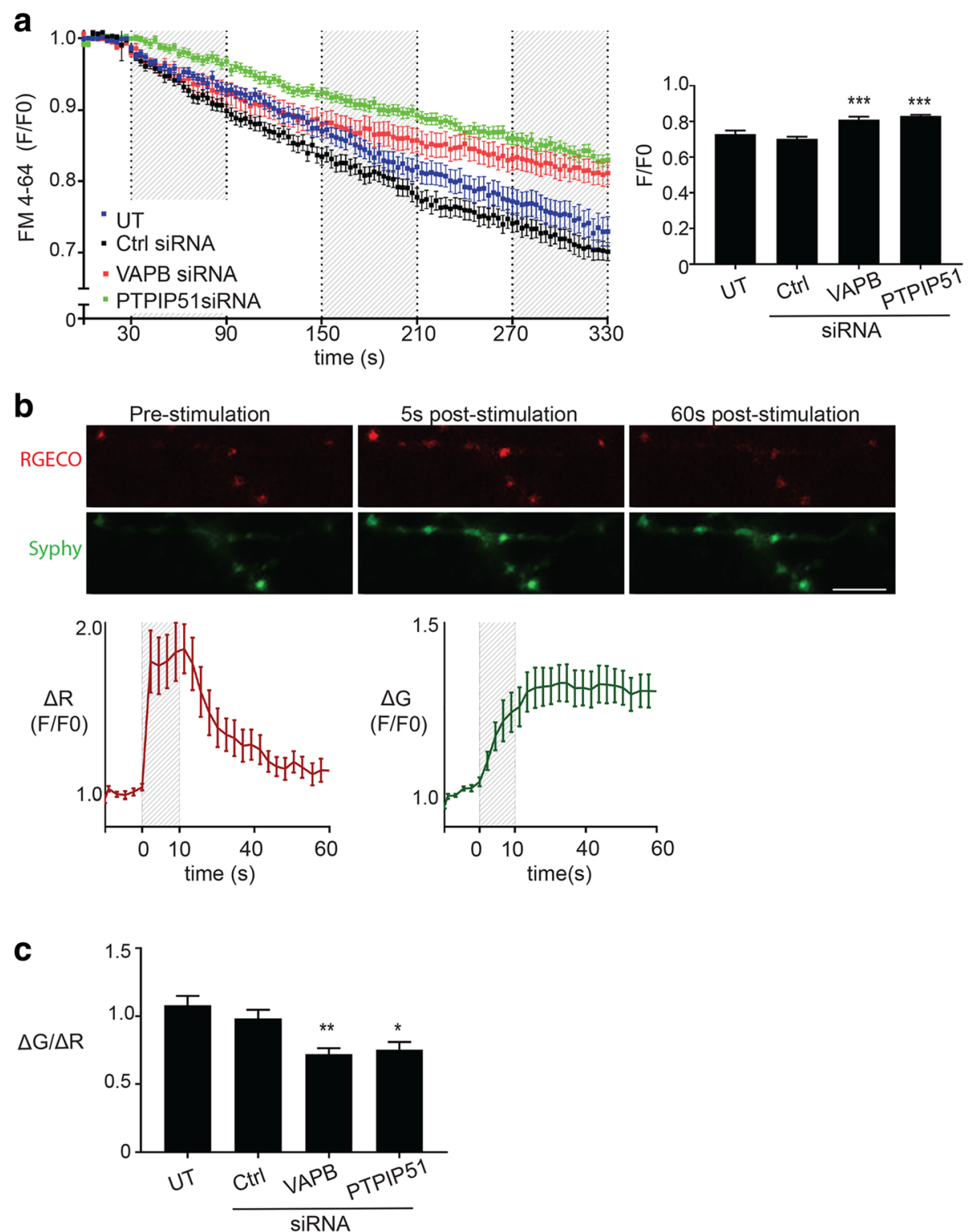

Fig. 4 Loss of VAPB or PTPIP51 disrupts synaptic activity. a Kinetics of FM 4-64 release from synaptic boutons in hippocampal neurons either untreated (UT) or treated with control (Ctrl), VAPB or PTPIP51 siRNAs. Neurons were loaded with FM 4-64 and synaptic activity induced by electrical field stimulation. Periods of electrical field stimulation are indicated by shaded regions. FM 4-64 signals were determined from images acquired by time-lapse microscopy. F/FO represents the ratio of the FM 4-64 fluorescent signals at each time point to signals at time 0 . Error bars are mean \pm SEM. Bar chart shows F/FO FM 4-64 fluorescent signals at time $330 \mathrm{~s}$. Data were analysed by one-way ANOVA with Tukey's post hoc test. $N=24$ boutons UT, $N=40$ boutons $C$ trl siRNA, $N=24$ boutons VAPB siRNA, $N=35$ boutons PTPIP51 siRNA from 3 independent experiments. Error bars are SEM, ${ }^{* * *} p \leq$ 0.001. b Representative images of transfected SypHy-RGECO axonal signals in neurons prior to and following electrical field stimulation to induce synaptic activity. Scale bar $=1.5 \mu \mathrm{m}$. Graphs show changes in SypHy $(\Delta \mathrm{G})$ and RGECO $(\Delta \mathrm{R})$ fluorescent signals; shading shows time of electrical field stimulation. $N=18$ synapses; error bars are SEM. c SypHy-RGECO reporter reveals reduced synaptic responses to electrical field stimulation in VAPB and PTPIP51 siRNA treated hippocampal neurons. Bar-graph shows the ratio between SypHy $(\Delta G)$ and RGECO $(\Delta R)$ fluorescence signals in response to electrical field stimulation. Data were analysed by one-way ANOVA and Tukey's post hoc test. $N=39$ synapses UT, $N=48$ synapses $C$ trl siRNA, $N=59$ synapses VAPB siRNA, $N=41$ synapses PTPIP51 siRNA from 3 to 4 independent experiments. Error bars are SEM; ${ }^{* *} p \leq 0.001$

ER-mitochondria tethers therefore have roles in both pre- and postsynaptic function. Others have recently shown that ER-mitochondria signaling can affect postsynaptic function although whether this involved the VAPB-PTPIP51 tethers was not reported [18]. Since synapse function is intimately related to both preand postsynaptic changes, these reductions in dendritic spine number induced by loss of VAPB and PTPIP51 may therefore influence some of the presynaptic changes we observe. 
The precise mechanisms by which the VAPB-PTPIP51 ER-mitochondria tethers affect synaptic function are not fully clear. However, dynamic changes in $\mathrm{Ca}^{2+}$ signaling are fundamental to synaptic transmission. Presynaptic $\mathrm{Ca}^{2+}$ levels regulate neurotransmitter release and dendritic $\mathrm{Ca}^{2+}$ alterations control synaptic plasticity [10, 40, $41,44]$. Synaptic transmission is also metabolically expensive and ATP production to drive this transmission is associated with mitochondrial $\mathrm{Ca}^{2+}$ levels since several dehydrogenases in the tricarboxylic acid cycle are $\mathrm{Ca}^{2+}$ regulated [12, 14, 15, 25, 34]. A primary function of ER-mitochondria contacts including those mediated by the VAPB-PTPIP51 tethering proteins is to facilitate delivery of $\mathrm{Ca}^{2+}$ to mitochondria from ER stores $[7,9,12$, $15,34,45]$. Indeed, the VAPB-PTPIP51 tethers have been linked to mitochondrial ATP production [13, 33, 46]. Our findings that the VAPB-PTPIP51 tethers regulate synaptic function are therefore consistent with the known roles of these proteins in $\mathrm{Ca}^{2+}$ homeostasis and the generation of mitochondrial ATP.

Aside from these roles, ER-mitochondria contacts also control a number of other fundamental cellular functions. For example, the contacts regulate lipid metabolism since the synthesis of some phospholipids involves precursor exchange between the two organelles and the tight contacts facilitate this exchange [7, 12, 34]. ER-mitochondria signaling involving the VAPB-PTPIP51 tethers also affects autophagosome formation [13]. Changes in lipid metabolism and autophagy are both known to influence synaptic function $[26,32]$. Thus, the VAPB-PTPIP51 tethers may also modulate synaptic function via their roles in these other processes.

Finally, PTPIP51 has been associated with changes in the activities of a number of signaling molecules and in particular, mitogen-activated protein kinase (MAP kinase) [4]. MAP kinase plays major roles in synaptic function [29]. In addition, and based upon its expression in different brain regions, PTPIP51 has been linked to learning and memory [5]. It will be interesting to determine whether the role of PTPIP51 and VAPB in regulating ER-mitochondria $\mathrm{Ca}^{2+}$ leads to downstream changes in MAP kinase and other signaling molecules.

As detailed above, damage to ER-mitochondria signaling has been associated with Alzheimer's disease, Parkinson's disease and FTD/ALS and for Parkinson's disease and FTD/ALS this can involves disruption of the VAPB\#PTPIP51 tethers [1, 23, 24, 33, 34, 45, 46]. Synaptic damage is a key feature of all these diseases but the mechanisms underlying this damage are not fully understood. Our findings that the VAPB-PTPIP51 tethers regulate synaptic activity therefore provide a novel route linking neurodegenerative disease insults with synaptic dysfunction. Future studies to determine whether such insults affect ER-mitochondria contacts and the VAPB-PTPIP51 tethers in synaptic regions would provide further insight into this topic. In addition, it will also be informative to determine whether correction of damaged VAPB-PTPIP51 tethers rescues synaptic damage. Interestingly, increasing ER-mitochondria contacts via overexpression of VAPB rescues $\alpha$-synuclein induced damage to mitochondrial $\mathrm{Ca}^{2+}$ levels [33]. Also, exogenous viral delivery of VAPB is protective in ALS mutant superoxide dismutase- 1 transgenic mice [22]. Thus, the findings we report here pave the way for future studies that address whether synaptic damage in neurodegenerative diseases is linked to changes in VAPB-PTPIP51 interactions and ER-mitochondria signaling.

\section{Conclusions}

Damage to ER-mitochondria signaling is now known to contribute to Alzheimer's disease, Parkinson's disease and FTD/ALS. For Parkinson's disease and FTD/ALS, this damage has been shown to involve disruption to the ER-mitochondria tethering proteins VAPB and PTPIP51 which function to recruit regions of ER to the mitochondrial surface. Loss of synaptic function is a key pathogenic feature of Parkinson's disease and FTD/ALS. Both ER and mitochondria are present in synaptic regions but whether ER-mitochondria signaling involving the VAPB-PTPIP51 tethers contributes to synaptic function is not known. Here we show that the VAPB-PTPIP51 tethering proteins are present and interact in synaptic regions and that loss of VAPB and PTPIP51 perturbs synaptic activity. Thus, damage to the VAPB-PTPIP51 tethers may contribute to synaptic dysfunction in Parkinson's disease and FTD/ALS.

\section{Additional files}

\section{Additional file 1: Movie 1. Electrical field stimulation of synaptic activity increases ER-mitochondria contacts. Movie shows SPLICS, fluorescent signals in hippocampal neurons prior to and after stimulation. Time in seconds (s) are shown. CB indicates cell body. Electrical field stimulation was applied at 30s. (MOV 10446 kb)}

Additional file 2: Movie 2. Electrical field stimulation of synaptic activity increases ER-mitochondria contacts including contacts close to synapses. Movie shows SPLICS signals (green) in hippocampal neuron processes loaded with FM 4-64 (red). Arrows show increased SPLICS signals closely associated with active synapses identified by loss of FM 4-64 signal. Time in seconds (s) are shown. Field stimulation was applied at 30s. (MOV 2534 kb)

\section{Abbreviations}

ALS: Amyotrophic lateral sclerosis; ANOVA: Analysis of variance; DIV: Days in vitro; ER: Endoplasmic reticulum; FTD: Frontotemporal dementia; GAPDH: Glyceraldehyde 3-phosphate dehydrogenase; IP3: Inositol 1,4,5-trisphosphate; MAM: Mitochondria associated ER membranes; MAP kinase: Mitogen activated protein kinase; MAP2: Microtubule-associated protein-2; NFH: Neurofilament heavy chain; PBS: Phosphate buffered saline; PDI: Protein disulphide isomerase; PLAs: Proximity ligation assays; PSD95: Post synaptic density protein-95; PTPIP51: Protein tyrosine phosphatase interacting protein-51; s: Seconds; SEM: Standard error of mean; SIM: Structured illumination microscopy; SN: Synaptoneurosomes; SPLICS: Split green fluorescent protein (GFP) ER-mitochondria contact reporter; TBS: Tris-HCl-buffered saline; TOM20: Translocase of the outer mitochondrial membrane protein-20; VAPB: Vesicle-associated membrane protein-associated protein B 


\section{Acknowledgements}

We thank the King's Nikon Imaging and Wohl Cellular Imaging Centres, and George Chennell for assistance in microscopy.

\section{Funding}

This work was supported by grants from the Alzheimer's Society (AlzSoc-287), Alzheimer's Research UK (ARUK-PG2014-5, ARUK-PG2017B-3, ARUK-EG2013B-1), UK Medical Research Council (MR/R022666/1) and Motor Neuron Disease Association (Gomez-Suaga/Oct17/967/799).

\section{Availability of data and materials}

Experimental tools and data are available from the corresponding author.

\section{Authors' contributions}

CCJM and PG-S designed the study and wrote the manuscript. CCIM, BP-N and WN designed and supervised experiments. PG-S performed most experiments and analyzed data. EG, DL, BP-N, GMM and SP performed experiments. TC and PP contributed reagents and expertise. All authors edited the manuscript. All authors read and approved the final manuscript.

\section{Ethics approval and consent to participate}

Not applicable.

\section{Consent for publication}

Not applicable.

\section{Competing interests}

The authors declare that they have no competing interests.

\section{Publisher's Note}

Springer Nature remains neutral with regard to jurisdictional claims in published maps and institutional affiliations.

\section{Author details}

'Department of Basic and Clinical Neuroscience, Maurice Wohl Clinical Neuroscience Institute, Institute of Psychiatry, Psychology and Neuroscience, King's College London, London SE5 9RX, UK. ²Department of Biomedical Sciences, University of Padova, Padova, Italy.

Received: 8 January 2019 Accepted: 24 February 2019

Published online: 06 March 2019

\section{References}

1. Area-Gomez E, Schon EA (2017) On the pathogenesis of Alzheimer's disease: the MAM hypothesis. FASEB J 31:864-867. https://doi.org/10.1096/fj. 201601309

2. Atluri PP, Ryan TA (2006) The kinetics of synaptic vesicle reacidification at hippocampal nerve terminals. J Neurosci 26:2313-2320. https://doi.org/10. 1523/jneurosci.4425-05.2006

3. Bae JR, Kim SH (2017) Synapses in neurodegenerative diseases. BMB Rep 50:237-246

4. Brobeil A, Dietel E, Gattenlohner S, Wimmer M (2017) Orchestrating cellular signaling pathways-the cellular "conductor" protein tyrosine phosphatase interacting protein 51 (PTPIP51). Cell Tissue Res 368:411-423. https://doi. org/10.1007/s00441-016-2508-5

5. Brobeil A, Viard M, Petri MK, Steger K, Tag C, Wimmer M (2015) Memory and PTPIP51--a new protein in hippocampus and cerebellum. Mol Cell Neurosci 64:61-73. https://doi.org/10.1016/j.mcn.2014.12.003

6. Cieri D, Vicario $M$, Giacomello $M$, Vallese F, Filadi $R$, Wagner T, Pozzan T, Pizzo P, Scorrano L, Brini M et al (2018) SPLICS: a split green fluorescent protein-based contact site sensor for narrow and wide heterotypic organelle juxtaposition. Cell Death Differ 25:1131-1145. https://doi.org/10. 1038/s41418-017-0033-z

7. Csordas G, Weaver D, Hajnoczky G (2018) Endoplasmic reticularmitochondrial Contactology: structure and signaling functions. Trends Cell Biol 28:523-540. https://doi.org/10.1016/j.tcb.2018.02.009

8. DaRocha-Souto B, Coma M, Perez-Nievas BG, Scotton TC, Siao M, SanchezFerrer P, Hashimoto T, Fan Z, Hudry E, Barroeta I et al (2012) Activation of glycogen synthase kinase-3 beta mediates beta-amyloid induced neuritic damage in Alzheimer's disease. Neurobiol Dis 45:425-437. https://doi.org/10. 1016/j.nbd.2011.09.002
9. De Vos KJ, Morotz GM, Stoica R, Tudor EL, Lau KF, Ackerley S, Warley A, Shaw CE, Miller CCJ (2012) VAPB interacts with the mitochondrial protein PTPIP51 to regulate calcium homeostasis. Hum Mol Genet 21:1299-1311. https://doi.org/10.1093/hmg/ddr559

10. Devine MJ, Kittler JT (2018) Mitochondria at the neuronal presynapse in health and disease. Nat Rev Neurosci 19:63-80. https://doi.org/10.1038/ nrn.2017.170

11. Ferreras S, Fernandez G, Danelon V, Pisano MV, Masseroni L, Chapleau CA, Krapacher FA, Mlewski EC, Masco DH, Arias C et al (2017) Cdk5 Is Essential for Amphetamine to Increase Dendritic Spine Density in Hippocampal Pyramidal Neurons. Front Cell Neurosci 11:372. https://doi.org/10.3389/fncel. 2017.00372

12. Filadi $R$, Theurey $P$, Pizzo P (2017) The endoplasmic reticulum-mitochondria coupling in heath and disease: molecules, functions and significance. Cell Calcium 62:1-15. https://doi.org/10.1016/j.ceca.2017.01.003

13. Gomez-Suaga P, Paillusson S, Stoica R, Noble W, Hanger DP, Miller CC (2017) The ER-mitochondria tethering complex VAPB-PTPIP51 regulates autophagy. Curr Biol 27:371-385. https://doi.org/10.1016/j.cub.2016.12.038

14. Griffiths EJ, Rutter GA (2009) Mitochondrial calcium as a key regulator of mitochondrial ATP production in mammalian cells. Biochim Biophys Acta 1787:1324-1333. https://doi.org/10.1016/j.bbabio.2009.01.019

15. Gutierrez T, Simmen T (2018) Endoplasmic reticulum chaperones tweak the mitochondrial calcium rheostat to control metabolism and cell death. Cell Calcium 70:64-75. https://doi.org/10.1016/j.ceca.2017.05.015

16. Hedskog L, Pinho CM, Filadi R, Ronnback A, Hertwig L, Wiehager B, Larssen P. Gellhaar S, Sandebring A, Westerlund M et al (2013) Modulation of the endoplasmic reticulum-mitochondria interface in Alzheimer's disease and related models. Proc Natl Acad Sci USA 110:7916-7921. https://doi.org/10. 1073/pnas.1300677110

17. Herms J, Dorostkar MM (2016) Dendritic spine pathology in neurodegenerative diseases. Annu Rev Pathol 11:221-250. https://doi.org/ 10.1146/annurev-pathol-012615-044216

18. Hirabayashi Y, Kwon SK, Paek H, Pernice WM, Paul MA, Lee J, Erfani P, Raczkowski A, Petrey DS, al PLA (2017) ER-mitochondria tethering by PDZD8 regulates $\mathrm{Ca} 2+$ dynamics in mammalian neurons. Science (New York, NY) 358:623-630. https://doi.org/10.1126/science.aan6009

19. Iwabuchi S, Kakazu Y, Koh JY, Goodman KM, Harata NC (2014) Examination of synaptic vesicle recycling using FM dyes during evoked, spontaneous, and miniature synaptic activities. J Vis Exp: Doi. https:// doi.org/10.3791/50557

20. Jackson RE, Burrone J (2016) Visualizing presynaptic calcium dynamics and vesicle fusion with a single genetically encoded reporter at individual synapses. Front Synaptic Neurosci 8:21. https://doi.org/10. 3389/fnsyn.2016.00021

21. Kasai H, Fukuda M, Watanabe S, Hayashi-Takagi A, Noguchi J (2010) Structural dynamics of dendritic spines in memory and cognition. Trends Neurosci 33:121-129. https://doi.org/10.1016/..tins.2010.01.001

22. Kim JY, Jang A, Reddy R, Yoon WH, Jankowsky JL (2016) Neuronal overexpression of human VAPB slows motor impairment and neuromuscular denervation in a mouse model of ALS. Hum Mol Genet 25 : 4661-4673. https://doi.org/10.1093/hmg/ddw294

23. Krols M, van Isterdael G, Asselbergh B, Kremer A, Lippens S, Timmerman V, Janssens S (2016) Mitochondria-associated membranes as hubs for neurodegeneration. Acta Neuropathol 131:505-523. https://doi.org/10.1007/ s00401-015-1528-7

24. Lau DHW, Hartopp N, Welsh NJ, Mueller S, Glennon EB, Morotz GM, Annibali A, Gomez-Suaga P, Stoica R, Paillusson S et al (2018) Disruption of ERmitochondria signalling in fronto-temporal dementia and related amyotrophic lateral sclerosis. Cell Death Disease 9:327. https://doi.org/10. 1038/s41419-017-0022-7

25. Laughlin SB, de Ruyter van Steveninck RR, Anderson JC (1998) The metabolic cost of neural information. Nat Neurosci 1:36-41. https://doi.org/10.1038/236.

26. Lauwers $E$, Goodchild R, Verstreken $P$ (2016) Membrane lipids in presynaptic function and disease. Neuron 90:11-25. https://doi.org/10.1016/j.neuron. 2016.02.033

27. Lazarenko RM, DelBove CE, Zhang Q (2018) Fluorescent measurement of synaptic activity using FM dyes in dissociated hippocampal cultured neurons. Bio Protoc 8. https://doi.org/10.21769/BioProtoc.2690

28. Ling SC, Polymenidou M, Cleveland DW (2013) Converging mechanisms in ALS and FTD: disrupted RNA and protein homeostasis. Neuron 79:416-438. https://doi.org/10.1016/j.neuron.2013.07.033 
29. Mao LM, Wang JQ (2016) Synaptically localized mitogen-activated protein kinases: local substrates and regulation. Mol Neurobiol 53:6309-6315. https://doi.org/10.1007/s12035-015-9535-1

30. Micheva KD, Taylor CP, Smith SJ (2006) Pregabalin reduces the release of synaptic vesicles from cultured hippocampal neurons. Mol Pharmacol 70: 467-476. https://doi.org/10.1124/mol.106.023309

31. Mironov SL, Symonchuk N (2006) ER vesicles and mitochondria move and communicate at synapses. J Cell Sci 119:4926-4934

32. Nikoletopoulou V, Tavernarakis N (2018) Regulation and roles of autophagy at synapses. Trends Cell Biol 28:646-661. https://doi.org/10. 1016/j.tcb.2018.03.006

33. Paillusson S, Gomez-Suaga P, Stoica R, Little D, Gissen P, Devine MJ, Noble W, Hanger DP, Miller CC (2017) Alpha-Synuclein binds to the ERmitochondria tethering protein VAPB to disrupt Ca2+ homeostasis and mitochondrial ATP production. Acta Neuropathol 134:129-149. https://doi. org/10.1007/s00401-017-1704-z

34. Paillusson S, Stoica R, Gomez-Suaga P, Lau DH, Mueller S, Miller T, Miller CC (2016) There's something wrong with my MAM; the ER-mitochondria axis and neurodegenerative diseases. Trends Neurosci 39:146-157. https://doi. org/10.1016/j.tins.2016.01.008

35. Perez-Nievas BG, Stein TD, Tai HC, Dols-Icardo O, Scotton TC, Barroeta-Espar I, Fernandez-Carballo L, de Munain EL, Perez J, Marquie M et al (2013) Dissecting phenotypic traits linked to human resilience to Alzheimer's pathology. Brain 136:2510-2526. https://doi.org/10.1093/brain/awt171

36. Pickett EK, Koffie RM, Wegmann S, Henstridge CM, Herrmann AG, ColomCadena M, Lleo A, Kay KR, Vaught M, Soberman R et al (2016) Non-Fibrillar oligomeric amyloid-beta within synapses. J Alzheimers Dis 53:787-800. https://doi.org/10.3233/jad-160007

37. Rieusset J (2018) Mitochondria-associated membranes (MAMs): an emerging platform connecting energy and immune sensing to metabolic flexibility. Biochem Biophys Res Commun 500:35-44. https://doi.org/10.1016/j.bbrc. 2017.06.097

38. Rother M, Brauner JM, Ebert K, Welzel O, Jung J, Bauereiss A, Kornhuber J, Groemer TW (2014) Dynamic properties of the alkaline vesicle population at hippocampal synapses. PLoS One 9:e102723. https://doi.org/10.1371/journal. pone.0102723

39. Rowland AA, Voeltz GK (2012) Endoplasmic reticulum-mitochondria contacts: function of the junction. Nat Rev Mol Cell Biol 13:607-625. https:// doi.org/10.1038/nrm3440

40. Segal M (2005) Dendritic spines and long-term plasticity. Nat Rev Neurosci 6:277-284. https://doi.org/10.1038/nrn1649

41. Sjostrom PJ, Rancz EA, Roth A, Hausser M (2008) Dendritic excitability and synaptic plasticity. Physiol Rev 88:769-840. https://doi.org/10.1152/physrev. 00016.2007

42. Soderberg O, Gullberg M, Jarvius M, Ridderstrale K, Leuchowius KJ, Jarvius J, Wester K, Hydbring P, Bahram F, al LLG (2006) Direct observation of individual endogenous protein complexes in situ by proximity ligation. Nat Methods 3:995-1000. https://doi.org/10.1038/nmeth947

43. Spires-Jones TL, Attems J, Thal DR (2017) Interactions of pathological proteins in neurodegenerative diseases. Acta Neuropathol 134:187-205. https://doi.org/10.1007/s00401-017-1709-7

44. Spruston N (2008) Pyramidal neurons: dendritic structure and synaptic integration. Nat Rev Neurosci 9:206-221. https://doi.org/10.1038/nrn2286

45. Stoica R, De Vos KJ, Paillusson S, Mueller S, Sancho RM, Lau KF, VizcayBarrena G, Lin WL, Xu YF, Lewis J et al (2014) ER-mitochondria associations are regulated by the VAPB-PTPIP51 interaction and are disrupted by ALS/ FTD-associated TDP-43. Nat Commun 5:3996. https://doi.org/10.1038/ ncomms4996

46. Stoica R, Paillusson S, Gomez-Suaga P, Mitchell JC, Lau DH, Gray EH, Sancho RM, Vizcay-Barrena G, De Vos KJ, Shaw CE et al (2016) ALS/FTD-associated FUS activates GSK-3beta to disrupt the VAPB-PTPIP51 interaction and ERmitochondria associations. EMBO Rep 17:1326-1342. https://doi.org/10. 15252/embr.201541726

47. Tai HC, Serrano-Pozo A, Hashimoto T, Frosch MP, Spires-Jones TL, Hyman BT (2012) The synaptic accumulation of hyperphosphorylated tau oligomers in Alzheimer disease is associated with dysfunction of the ubiquitinproteasome system. Am J Pathol 181:1426-1435. https://doi.org/10.1016/j. ajpath.2012.06.033

48. Tyler WJ, Zhang XL, Hartman K, Winterer J, Muller W, Stanton PK, PozzoMiller $L$ (2006) BDNF increases release probability and the size of a rapidly recycling vesicle pool within rat hippocampal excitatory synapses. J Physiol 574:787-803. https://doi.org/10.1113/jphysiol.2006.111310

49. Vlachos A, Korkotian E, Schonfeld E, Copanaki E, Deller T, Segal M (2009) Synaptopodin regulates plasticity of dendritic spines in hippocampal neurons. J Neurosci 29:1017-1033. https://doi.org/10.1523/ jneurosci.5528-08.2009

50. Wu Y, Whiteus C, Xu CS, Hayworth KJ, Weinberg RJ, Hess HF, De Camilli P (2017) Contacts between the endoplasmic reticulum and other membranes in neurons. Proc Natl Acad Sci U S A 114:E4859-E4867. https://doi.org/10. 1073/pnas.1701078114

\section{Ready to submit your research? Choose BMC and benefit from:}

- fast, convenient online submission

- thorough peer review by experienced researchers in your field

- rapid publication on acceptance

- support for research data, including large and complex data types

- gold Open Access which fosters wider collaboration and increased citations

- maximum visibility for your research: over $100 \mathrm{M}$ website views per year

At BMC, research is always in progress.

Learn more biomedcentral.com/submissions 